\title{
A New Approach to Epistemic Logic
}

\author{
Giovanna Corsi - Gabriele Tassi \\ Dipartimento di Filosofia - Università di Bologna \\ via Zamboni, 38 - I-40126 Bologna \\ giovanna.corsi@unibo.it \\ Università di Bologna \\ gabriele.tassi@studio.unibo.it
}

May 30, 2013

\begin{abstract}
A new language for epistemic logic is introduced in which the epistemic operators are of the form $\left|x: x_{1} \ldots x_{n}\right|$ with the intended reading

$$
\text { " } x \text { knows of } x_{1} \ldots x_{n} \text { that } \ldots " .
$$

Analogously we can express " $t$ knows of $t_{1} \ldots t_{n}$ that $\ldots$ ", where $t, t_{1} \ldots t_{n}$ are terms. An advantage of this approach is that we can quantify on the agents, "every $y$ knows of $x_{1} \ldots x_{n}$ that $A$ " or "some expert knows of $t_{1} \ldots t_{n}$ that $A$ " can easily be expressed. The semantics we present for this language is a generalization of the transition semantics, called epistemic transition semantics in which the possible worlds are states of affairs compatible with the epistemic state of some agent. A calculus is presented and shown to be complete with respect to epistemic transition semantics.
\end{abstract}

\section{Introduction}

Reasoning about knowledge by the help of logical notions and tools has originated a mess of different approaches to knowledge depending, among other things, on the intended applications: ordinary language, artificial intelligence, game theory, comunication protocols. Various types of logics have 
been introduced starting with epistemic logics in the style of Hintikka [6], then multi-agent logics and common knowledge logics in the style of Fagin et al.,[4]. This last book has set the agenda for future research up to the present days and this paper locates itself in its wake.

Typically, the first step of every approach considered consists in setting the appropriate language in order to deal with the chosen aspect or variant of the notion of knowledge under study. As a matter of fact most of the languages are propositional languages obtained by adding to the boolean connectives a finite set of modal operators. In the case of epistemic logic these operators are indexed by agents $K_{i}, K_{j}, \ldots$

$$
K_{i}(A)
$$

agent $i$ knows that $A$

When we move to first-order level, quantification is allowed with respect to $A$ but not with respect to the agents, we can say that ' $i$ knows that someone is $P$ ', but not that 'someone knows that someone is $P$ '.

We will take a quite different approach by introducing epistemic operators indexed by terms analogous to the indexed modal operators for alethic modalities. In the case of alethic modalities, see [2], $\square P(x)$ is not a wellformed formula since $x$ is free in $P(x)$ and it has to be replaced by

$$
|x| P(x)
$$

to be read as

'it is necessary for $x$ to be $P(x)$ '.

$|x|$ is a box-operator indexed by $x$. A more complex form of the box-operator is the following one

$$
\left|\begin{array}{l}
i \\
x
\end{array}\right| P(x)
$$

'it is necessary for the individual $i$ to have the property $\lambda x . P(x)$ '.

Dually,

$$
\left\langle\begin{array}{l}
i \\
x
\end{array}\right\rangle P(x)
$$

' it is possible for $i$ to have the property $\lambda x . P(x)$ '. Again,

$$
\left|\begin{array}{ll}
i & j \\
x & y
\end{array}\right| R(x, y)
$$


'it is necessary for $i$ and $j$ to stand in the relation $\lambda x \lambda y \cdot R(x, y)$ '.

In the case of epistemic modalities we need to distinguish the agent of the act of knowing from the objects of knowledge, therefore epistemic operators will have the form

$$
\left|t:{ }_{x_{1}}^{t_{1}} \cdots{ }_{x_{n}}^{t_{n}}\right| A
$$

$t$ knows of $t_{1} \ldots t_{n}$ that $A$.

where $x_{1} \ldots x_{n}$ is a list of variables without repetitions that may contain also variables occurring in $t$, and $A$ contains at most the variables $x_{1} \ldots x_{n}$. Features of the notation just introduced:

- the epistemic operator binds the variables $x_{1}, \ldots, x_{n}$ occurring in $A$

- the variables occurring in $t, t_{1}, \ldots, t_{n}$ are the free variables of $\left|t:{ }_{x_{1}}^{t_{1}} \ldots{ }_{x_{n}}^{t_{n}}\right| A$.

- if $A$ is a sentence $|t:| A$ is well formed, ' $t$ knows that $A$ '

- by convention $\left|x: x_{1} \ldots x_{n}\right| A$ stands for $\left|x:{ }_{x_{1}}^{x_{1}} \ldots{ }_{x_{n}}^{x_{n}}\right| A$

- de re / de dicto distinction de re $\quad\left|t:{ }_{x}^{i}\right| P x \quad$ ' $t$ knows of $i$ that (s)he is $\mathrm{P}$ ' de dicto $\quad|t:| P i \quad$ ' $t$ knows that $P i$ '

- substitution is indicated inside the epistemic operator, it is not carried out in $A$

$$
\begin{gathered}
\left(\left|x: x_{1} \ldots x_{n}\right| A\right)\left[t / x, t_{1} / x_{1} \ldots t_{n} / x_{n}\right]:=\left|t:{ }_{x_{1}}^{t_{1}} \ldots{ }_{x_{n}}^{t_{n}}\right| A \\
\left(\left|t:{ }_{x_{1}}^{t_{1}} \ldots{ }_{x_{n}}^{t_{n}}\right| A\right)[s / y]:=\left|{ }_{x}^{t[s / y]}:{ }_{x_{1}}^{t_{1}[s / y]} \ldots{ }_{x_{n}}^{t_{n}[s / y]}\right| A
\end{gathered}
$$

- substitution does not commute with epistemic operators.

$$
\left|t:{ }_{x}^{i}\right| P x \not>|t:| P i
$$

We need to add specific axioms if we want substitution to commute with epistemic-operators. 
Before giving the formal definition of a first-order epistemic language with indexed knowledge operators, let us look at some examples.

All Mary's friends know that she likes Paul

$$
\forall x\left(\operatorname{Friend}(x, \text { Mary }) \rightarrow\left|x:{ }_{y}^{\text {Mary }}\right| \operatorname{LIKES}(y, \text { Paul })\right)
$$

and this sentence is not equivalent to

$$
\forall x(\operatorname{Friend}(x, \text { Mary }) \rightarrow|x:| \operatorname{LikES}(\text { Mary, Paul }))
$$

In the latter sentence Mary is in a de dicto position, in the former sentence in a de re position.

Someone knows that all Peter's friends know that he likes Mary

$$
\exists x\left|x:{ }_{y}^{\text {Peter }}\right| \forall z(\operatorname{FRIEND}(z, y) \rightarrow|z: y| \operatorname{LIKES}(y, \text { Mary }))
$$

Someone knows who is late

$$
\exists x \exists y|x: y| \operatorname{LATE}(y)
$$

Someone knows who Dr Smith is

$$
\exists x \exists y|x: y|(y=\text { Dr Smith })
$$

Peter knows that he is Peter

$$
\text { |Peter }:{ }_{x}^{\text {Peter }} \mid(x=\text { Peter })
$$

All experts known by Peter know that smoking is dangerous

$$
\forall x(\operatorname{EXPERT}(x) \wedge \exists y \mid \text { Peter }: x, y|(x=y) \rightarrow| x: \mid \text { DANGERous }(\text { smoking }))
$$

\section{Language}

\section{Definition 2.1}

- Terms are either variables or individual constants and the set of free variables occurring in a term $t, f v(t)$, is either $\{t\}$ if $t$ is a variable or the empty set, otherwise. 
- The logical symbols are $\perp, \rightarrow, \forall,\left|t:{ }_{x_{1}}^{t_{1}} \ldots{ }_{x_{n}}^{t_{n}}\right|, n \geq 0$, where $x_{1}, \ldots, x_{n}$ is a list of pairwise distinct variables and $t, t_{1}, \ldots, t_{n}$ are terms. When $n=0$ we write $|t:|$.

Definition 2.2 of well formed formula and of free variable in a wff. wff free variables

$$
\begin{array}{ll}
\perp & f v(\perp)=\emptyset \\
P^{n} t_{1}, \ldots, t_{n} & f v\left(P^{n} t_{1}, \ldots, t_{n}\right)=f v\left(t_{1}\right) \cup \ldots \cup f v\left(t_{n}\right) \\
A \rightarrow B & f v(A \rightarrow B)=f v(A) \cup f v(B) \\
\left|t:{ }_{x_{1}}^{t_{1}} \ldots{ }_{x_{n}}^{t_{n}}\right| A & \text { where } f v(A) \subseteq\left\{x_{1}, \ldots, x_{n}\right\} \\
& f v\left(\left|t:{ }_{x_{1}}^{t_{1}} \ldots{ }_{x_{n}}^{t_{n}}\right| A\right)=f v(t) \cup f v\left(t_{1}\right) \cup \ldots \cup \\
& f v\left(t_{n}\right) \\
\forall x A & f v(\forall x A)=f v(A)-\{x\}
\end{array}
$$

$\neg A, A \vee B, A \wedge B, A \leftrightarrow B, \exists x A,\left\langle t:{ }_{x_{1}}^{t_{1}} \ldots{ }_{x_{n}}^{t_{n}}\right\rangle A$ are defined as usual and $\left|x: x_{1} \ldots x_{n}\right| A$ and $\left\langle x: x_{1} \ldots x_{n}\right\rangle A$ stand for $\left|x: x_{x_{1}}^{x_{1}} \ldots{ }_{x_{n}}^{x_{n}}\right| A$ and $\left\langle x:{ }_{x_{1}}^{x_{1}} \ldots{ }_{x_{n}}^{x_{n}}\right\rangle A$, respectively.

Definition 2.3 of simultaneous substitution. Given a wff A containing the free variables $x_{1}, \ldots, x_{k}$, we define the wff $A\left[s_{1} / x_{1} \ldots s_{k} / x_{k}\right]$ where the term $s_{i}$ is substituted for $x_{i}, 1 \leq i \leq k$. Let $[\mathbf{s} / \mathbf{x}]={ }_{d f}\left[s_{1} / x_{1} \ldots s_{k} / x_{k}\right]$.

- $\perp[\mathbf{s} / \mathbf{x}]=\perp$

- $\left(P^{n} t_{1}, \ldots, t_{n}\right)[\mathbf{s} / \mathbf{x}]=P^{n}\left(t_{1}[\mathbf{s} / \mathbf{x}], \ldots, t_{n}[\mathbf{s} / \mathbf{x}]\right)$, where

$-t_{i}[\mathbf{s} / \mathbf{x}]=s_{i} \quad$ if $\quad t_{i} \in\left\{x_{1}, \ldots, x_{k}\right\}$

$-t_{i}[\mathbf{s} / \mathbf{x}]=t_{i} \quad$ if $\quad t_{i} \notin\left\{x_{1}, \ldots, x_{k}\right\}$

- $(A \rightarrow B)[\mathbf{s} / \mathbf{x}]=(A[\mathbf{s} / \mathbf{x}] \rightarrow B[\mathbf{s} / \mathbf{x}])$ 
- $(\forall y A)[\mathbf{s} / \mathbf{x}]=$

$$
= \begin{cases}\forall y(A[\mathbf{s} / \mathbf{x}]) & \text { if } y \notin\left(\left\{x_{1}, \ldots, x_{k}\right\} \cup\left\{s_{1}, \ldots, s_{k}\right\}\right) \\ \forall z((A[z / y])[\mathbf{s} / \mathbf{x}] & \text { where } z \text { doesn't occur in } \forall y A \text { and } z \notin\left\{s_{1}, \ldots, s_{k}\right\} \\ & \text { if } y \notin\left\{x_{1}, \ldots, x_{k}\right\} \text { and } y \in\left\{s_{1}, \ldots, s_{k}\right\} \\ \forall y A & \text { if } y \in\left\{x_{1}, \ldots, x_{k}\right\}\end{cases}
$$

- $\left(\left|t:{ }_{y_{1}}^{t_{1}} \cdots{ }_{y_{n}}^{t_{n}}\right| A\right)[\mathbf{s} / \mathbf{x}]=\left|t[\mathbf{s} / \mathbf{x}]:{ }_{y_{1}}^{t_{1}[\mathbf{s} / \mathbf{x}]} \cdots{ }_{y_{n}}^{t_{n}}[\mathbf{s} / \mathbf{x}]\right| A$

\section{Semantics}

The main idea behind the epistemic transition semantics is that

$$
\left|t:{ }_{x}^{s}\right| P(x)
$$

is true at a world $w$ if $t$ is an individual existing at $w, s$ is an individual existing at $w$ and in all worlds compatible with the epistemic state of $t$ the $t$-counterparts of $s$ (the counterparts of $s$ according to $t$ ) in those worlds satisfy $P(x)$.

$$
|t:| P(s)
$$

is true at a world $w$ if $t$ is an individual existing at $w$ and in all worlds compatible with the epistemic state of $t$ whoever is $s$ in those worlds satisfies $P(x)$.

An epistemic transition model (in brief, an epistemic model) is a family of classical models endowed with (1) a relation of compatibility between individuals and models and (2) a counterpart relation between individuals of different models or of the same model. We will call worlds the classical models, following the terminology of possible world semantics. In details, let $W$ be a not empty set of worlds, so each $w \in W$ is a pair $\left\langle D_{w}, I_{w}\right\rangle$ where $D_{w}$ is a not-empty set, the domain of $w$ and $I_{w}$ is an interpretation function such that:

- for every relation $P^{n}, I_{w}\left(P^{n}\right) \subseteq\left(D_{w}\right)^{n}$

- $I_{w}(=)=\left\{\langle a, a\rangle: a \in D_{w}\right\}$

- for every individual constant $i, I_{w}(i) \in D_{w}$ 
We assume that $D_{w} \cap D_{v}=\emptyset$ when $w \neq v$. By $\prec$ we denote a relation between elements of $\mathcal{E}=\bigcup\left\{D_{w}\right\}_{w \in W}$ and elements of $W$ :

$$
\prec \subseteq(\mathcal{E} \times \mathcal{W})
$$

If $a \prec v$ holds, then we say that the world $v$ is epistemically compatible with the individual $a$ or that $v$ is compatible with the epistemic state of $a$. By $\stackrel{a}{\longmapsto}$ we denote the counterpart relation parametrized by the individual $a$ :

$$
\stackrel{a}{\longrightarrow} \subseteq \bigcup\left\{D_{w} \times D_{v}: a \in D_{w} \wedge a \prec v\right\}
$$

If $a, b \in D_{w}, c \in D_{v}$ and $b \stackrel{a}{\longmapsto} c$ holds, then we say that $c$ is a counterpart of $b$ according to $a$ (in a world epistemically compatible with $a$ ).

Definition 3.1 An epistemic transition model $\mathcal{M}=\langle W, \prec, \longmapsto, D, I\rangle$ is a quintuple where $W$ and $\prec$ are defined as above, $\longmapsto=\bigcup\{\stackrel{a}{\longmapsto}\}_{a \in \mathcal{E}}, D$ is a function that associates to any $w \in W$ its domain $D_{w}$ and $I$ is a function that associates to any $w \in W$ its interpretation function $I_{w}$.

Definition 3.2 For every $w \in W$, a $w$-assignment is a function $\sigma: V A R \rightarrow$ $D_{w}$. If $\sigma$ is a $w$-assignment, $\sigma^{x \triangleright d}$ denotes the $w$-assignment which behaves exactly like $\sigma$ except that it maps $x$ to $d \in D_{w}$.

Given a $w$-assignment $\sigma$ the interpretation of $t$ in $w$ under $\sigma, I_{w}^{\sigma}(t)$, is defined in the standard way:

- $I_{w}^{\sigma}(x)=\sigma(x)$

- $I_{w}^{\sigma}(i)=I_{w}(i)$

Notational convention. When no ambiguity can arise, we write $\sigma(t)$ instead of $I_{w}^{\sigma}(t)$.

Definition 3.3 of satisfaction. We define when a wff $A$ is satisfied at $w$ by a w-assignment $\sigma$ in an epistemic model $\mathcal{M}, \sigma \models_{w}^{\mathcal{M}} A$.

$$
\begin{array}{rlrl}
\sigma & \models_{w}^{\mathcal{M}} \perp \\
\sigma & \models_{w}^{\mathcal{M}} P^{k}\left(t_{1} \ldots t_{k}\right) \quad \text { iff } \quad \sigma\left(t_{1}, \ldots, t_{k}\right) \in I_{w}\left(P^{k}\right) \\
\sigma & \models_{w}^{\mathcal{M}} B \rightarrow G & \text { iff } \quad \sigma \forall_{w}^{\mathcal{M}} B \text { or } \sigma \models_{w}^{\mathcal{M}} G \\
\sigma & \models_{w}^{\mathcal{M}} \forall x G & \text { iff } \quad \text { for all } d \in D_{w}, \sigma^{x \triangleright d} \models_{w}^{\mathcal{M}} G
\end{array}
$$


$\sigma \models{ }_{w}^{\mathcal{M}}\left|t:{ }_{y_{1}}^{t_{1}} \ldots{ }_{y_{n}}^{t_{n}}\right| G \quad$ iff $\quad$ for all $v$ such that $\sigma(t) \quad \prec \quad v$, and all v-assignments $\tau$ such that $\sigma\left(t_{1}\right) \stackrel{\sigma(t)}{\longmapsto}$ $\tau\left(y_{1}\right), \ldots, \sigma\left(t_{n}\right) \stackrel{\sigma(t)}{\longmapsto} \tau\left(y_{n}\right)$, then $\tau \models_{v}^{\mathcal{M}} G$

\section{Definition 3.4 .}

- $A$ wff $A$ is true at $w$ in $\mathcal{M}, \models_{w}^{\mathcal{M}} A$, iff for every $w$-assignment $\sigma$, $\sigma \mid={ }_{w}^{\mathcal{M}} A$.

- A wff $A$ is true in $\mathcal{M}, \models^{\mathcal{M}} A$, iff for every $w, \models_{w}^{\mathcal{M}} A$.

- A wff $A$ is valid on a class $C$ of epistemic transition models iff $A$ is true in each of them.

Lemma 3.5 on substitution and satisfaction for terms and formulas. Let $\sigma$ be a w-assignment.

$$
\begin{gathered}
\sigma(t[s / x])=\sigma^{x \triangleright \sigma(s)}(t) \\
\sigma \models_{w} A[s / x] \quad \text { iff } \quad \sigma^{x \triangleright \sigma(s)} \models_{w} A
\end{gathered}
$$

Proof By induction on $A$.

- $A=P^{n}\left(t_{1}, \ldots, t_{n}\right)$

$\sigma^{x \triangleright \sigma(s)}==_{w} P^{n}\left(t_{1}, \ldots, t_{n}\right)$ iff $\left\langle\sigma^{x \triangleright \sigma(s)}\left(t_{1}\right), \ldots, \sigma^{x \triangleright \sigma(s)}\left(t_{n}\right)\right\rangle \in I_{w}\left(P^{n}\right)$ iff $\left\langle\sigma\left(t_{1}[s / x]\right), \ldots, \sigma\left(t_{n}[s / x]\right)\right\rangle \in I_{w}\left(P^{n}\right)$ iff $\sigma \models_{w} P^{n}\left(t_{1}[s / x], \ldots, t_{n}[s / x]\right)$ iff $\sigma \models{ }_{w} P^{n}\left(t_{1}, \ldots, t_{n}\right)[s / x]$.

- $A=\forall y B$ and $y \neq s$ and $y \neq x$ $\sigma^{x \triangleright \sigma(s)} \models_{w} \forall y B$ iff for all $d \in D_{w}, \sigma^{x \triangleright \sigma(s), y \triangleright d} \models_{w} B$ iff for all $d \in D_{w}$, $\sigma^{y \triangleright d, x \triangleright \sigma(s)} \models_{w} B$ iff by induction hypothesis for all $d \in D_{w}, \sigma^{y \triangleright d} \models_{w}$ $B[s / x]$ iff $\sigma \models_{w} \forall y(B[s / x])$ iff by def. of substitution $\sigma \models_{w}(\forall y B)[s / x]$.

The cases in which either $y=s$ or $y=x$ are similar.

- $A=\left|{ }_{y_{1}}^{t_{1}} \ldots y_{y_{n}}^{t_{n}}\right| B$ $\sigma^{x \triangleright \sigma(s)} \models{ }_{w}\left|t::_{y_{1}}^{t_{1}} \ldots{ }_{y_{n}}^{t_{n}}\right| B$ iff

$\tau \models{ }_{v} B$ for all $v$-assignment $\tau$ such that $\sigma^{x \triangleright \sigma(s)}\left(t_{i}\right) \stackrel{\sigma^{x \triangleright \sigma(s)}(t)}{\longmapsto} \tau\left(y_{i}\right)$, $1 \leq i \leq n$, iff 


$$
\begin{aligned}
& \tau={ }_{v} B \text { for all } v \text {-assignment } \tau \text { such that } \sigma\left(t_{i}[s / x]\right) \stackrel{\sigma(t[s / x])}{\longmapsto} \tau\left(y_{i}\right), 1 \leq \\
& i \leq n \text {, iff } \\
& \sigma\left|=_{w}\right| t[s / x],{ }_{y_{1}}^{t_{1}[s / x]} \ldots y_{n}^{t_{n}[s / x]} \mid B \text { iff } \\
& \sigma={ }_{w}\left(\left|t,{ }_{y_{1}}^{t_{1}} \ldots{ }_{y_{n}}^{t_{n}}\right| B\right)[s / x] \text {. }
\end{aligned}
$$

\section{$3.1 \quad$ Validity}

The epistemic semantics we have seen so far is a generalization of the transition semantics presented in Corsi [2] and at the same time a particular case of a more general semantics called cone transition semantics due to Gabriele Tassi [?] and [3]. Most of the results proved in [2] hold for the epistemic case. The main difference with respect to transition semantics is that the accessibility relation among worlds is parametrized by individuals. We do not say anymore that a world $w$ is related to or accessible to another world $v$, but rather that $v$ is compatible with the epistemic state of an individual $a$ living in $w$. Moreover, as we have seen, also the counterpart relation is parametrized by individuals, so we speak of the $a$-counterpart of $b$, meaning the counterpart of $b$ according to $a$, parametrized by $a$.

Notice first that no condition has been put in order to establish some connections between the counterparts in a world $v$ of an individual $b$ living in $w$ and the interpretation of $b$ in $v$. This fact has the consequence that the following two types of knowledge are quite different:

$$
\begin{gathered}
\left|i::_{x_{1} x_{2}}^{t} x_{1}\right|\left(x_{1}=x_{2}\right) \quad i \text { knows of } t \text { and } s \text { that they are equal } \\
|i:|(t=s) \quad i \text { knows that } t \text { is equal to } s
\end{gathered}
$$

The first sentence is true at a world $w$ iff in all worlds $v$ compatible with the epistemic state of $\underline{i}$, all the $\underline{i}$-counterparts in $v$ of $\underline{t}$ and $\underline{s}$ (the interpretation of $t$ and $s$ in $w$ ) are identical. The second sentence is true at $w$ iff in all worlds $v$ compatible with the epistemic state of $\underline{i}$ the interpretation of $t$ and $s$ in $v$ are identical.

For particular individual constants $i, t$ and $s$ we can assume that the $\underline{i}$-counterparts in a world $v$ of $\underline{t}$ in $w$ include the interpretation of $t$ in $v$. A consequence is that the wff

$$
\left|i: \begin{array}{ll}
t & s \\
x_{1} & x_{2}
\end{array}\right|\left(x_{1}=x_{2}\right) \rightarrow|i:|(t=s)
$$

is valid. When this is the case we say that the terms $t$ and $s$ are $i$-rigid, i.e. are rigid terms from the point of view of $i$. For some student $i$ it might 
well be that if (s)he knows of Walter Scott and Ivanhoe that the first is the author of the second, than (s)he knows also the fact that Walter Scott is the author of Ivanhoe, because in the worlds (s)he can envisage the counterparts of both Walter Scott and Ivanhoe include the interpretations of both names in those worlds.

We can impose even stronger constrains on the counterpart relation, e.g. that the $\underline{i}$-counterpart in a world $v$ of the interpretations in $w$ of $t$ and $s$ coincide with the interpretations of $t$ and $s$, respectively, in $v$. For example if $t$ and $s$ are numbers, say 9 and 7, we may want that for any individual $i$, the $\underline{i}$-counterpart in a world $v$ of the interpretations of 9 and 7 in $w$ coincide with the interpretation of 9 and 7 in $v$, respectively. When this is the case the terms 9 and 7 are said to be $i$-stable and the following formula is valid

$$
\left|i: \begin{array}{cc}
9 & 7 \\
x_{1} x_{2}
\end{array}\right| A\left(x_{1}, x_{2}\right) \leftrightarrow|i:| A(9,7)
$$

This equivalence doesn't hold in general, not even for variables, instead the following implication, say from de re to de dicto, holds for variables:

$$
R G_{e}^{v} \quad\left|t:{ }_{x_{1}}^{y_{1}} \ldots{ }_{x_{n}}^{y_{n}}\right| A \rightarrow\left|y: v_{1} \ldots v_{k}\right|\left(A\left[y_{1} / x_{1} \ldots y_{n} / x_{n}\right]\right)
$$

where $v_{1} \ldots v_{k}$ are the variables $y_{1} \ldots y_{n}$ without repetitions.

Therefore we say that variables are rigid designators. In the case of aletic modalities it is often assumed that all terms, not just variables, are rigid designators and so the following formula is taken as an axiom

$R G \quad\left|{ }_{x_{1}}^{t_{1}} \ldots{ }_{x_{n}}^{t_{n}}\right| A \rightarrow\left|v_{1} \ldots v_{k}\right|\left(A\left[t_{1} / x_{1} \ldots t_{n} / x_{n}\right]\right)$

where $v_{1} \ldots v_{k}$ are the variables occurring in $t_{1}, \ldots t_{n}$.

The rigidity axiom is untenable, in general, in the epistemic case:

$$
R G_{e} \quad\left|t:{ }_{x_{1}}^{t_{1}} \ldots{ }_{x_{n}}^{t_{n}}\right| A \rightarrow\left|v_{1} \ldots v_{k}\right| y:\left(A\left[t_{1} / x_{1} \ldots t_{n} / x_{n}\right]\right)
$$

Let us stress that the converse of $R G_{e}^{v}$ is not valid, just consider the following instance:

$$
|i: y|(y=y) \rightarrow\left|i: \underset{x_{1} x_{2}}{y}{ }^{y}\right|\left(x_{1}=x_{2}\right)
$$

It is certainly true that in all worlds compatible with the epistemic state of $\underline{i}$, each individual is identical with itself, but at the same time if $y$ has two different $\underline{i}$-counterparts in a world $v$, then $\left(x_{1}=x_{2}\right)$ may be falsified in $v$.

\section{The epistemic logic Q.K $K_{e}$}

Now we present a calculus for epistemic logic which makes no assumptions either on the compatibility relation or on the counterpart relation. Q. $K_{e}$ 
intends to be the core system of any quantified logic either of belief or of knowledge or of obligation. We can think of weaker systems than $Q . K_{e}$ in the style of Gabriele Tassi [7], where the greater generality of Tassi's systems resides in the fact that the epistemic operators are indexed by lists of terms and not by pairs composed of a term and a set of terms, as we do, see axiom PRM.

Here are the axioms and inference rules of $Q . K_{e}$.

Tautologies

$$
\begin{array}{ll}
\mathrm{PRM}_{e} & \left|x: x_{1} \ldots x_{n}\right| A \leftrightarrow\left|x: x_{i_{1}} \ldots x_{i_{n}}\right| A \\
& \text { for every permutation } x_{i_{1}} \ldots x_{i_{n}} \text { of } x_{1} \ldots x_{n} \\
\mathrm{~K}_{e} \quad & \left|x: x_{1} \ldots x_{n}\right|(A \rightarrow B) \rightarrow\left(\left|x: x_{1} \ldots x_{n}\right| A \rightarrow\left|x: x_{1} \ldots x_{n}\right| B\right) \\
\mathrm{UI} & \forall x A(x) \rightarrow A \\
\mathrm{LNGT}_{e} & \left|x: x_{1} \ldots x_{n}\right| A \rightarrow\left|x: x_{1} \ldots x_{n}, x_{n+1}\right| A \\
R G_{e}^{v} \quad\left|y:{ }_{x_{1}}^{y_{1}} \ldots x_{x_{n}}^{y_{n}}\right| A \rightarrow\left|y: v_{1} \ldots v_{k}\right|\left(A\left[y_{1} / x_{1} \ldots y_{n} / x_{n}\right]\right)
\end{array}
$$

where $v_{1} \ldots v_{k}$ are the variables $y_{1} \ldots y_{n}$ without ripetitions.

ID $\quad x=x$

$\mathrm{LBZ} \quad t=s \rightarrow(A[t / x] \rightarrow A[s / x])$

$$
\frac{A \quad A \rightarrow B}{B}(M P)
$$

$$
\begin{aligned}
& \frac{A}{\left|x: x_{1} \ldots x_{n}\right| A} \quad\left(N_{e}\right) \quad \text { provided }\left\{x_{1}, \ldots, x_{n}\right\} \supseteq f v(A) . \\
& \frac{A \rightarrow B}{A \rightarrow \forall x B} \quad(U G) \quad \text { provided } x \notin f v(A)
\end{aligned}
$$




\section{$\frac{A}{A[s / x]} \quad(S F V)$}

The notions of proof and theorem are defined in the usual way.

\section{Completeness of $Q . K_{e}$}

The completeness proof we present follows the same strategy of the proof given in Corsi [2] and in Ghilardi [1]. Given a language with indexed operators $\mathcal{L}$, we define a classical first-order language $\overline{\mathcal{L}}$ which contains the same predicate and constant symbols of $\mathcal{L}$, and moreover for each formula $\left|t: x_{1} \ldots x_{n}\right| A$ of $\mathcal{L}$ a new predicate symbol $P_{\left|: x_{1} \ldots x_{n}\right| A}^{n+1}$. Then we translate each formula of $\mathcal{L}$ into a formula of $\overline{\mathcal{L}}$ according to the following definition:

\section{Definition 5.1}

$$
\begin{array}{ll}
\bar{\perp} & =\perp \\
\overline{P^{n}\left(t_{1}, \ldots, t_{n}\right)} & =P^{n}\left(t_{1}, \ldots, t_{n}\right) \\
\overline{s=t} & =s=t \\
\overline{(A \rightarrow B)} & =\bar{A} \rightarrow \bar{B} \\
\overline{\forall x_{i} A} & =\forall x_{i} \bar{A} \\
\overline{\left|t: t_{x_{1}} \ldots x_{x_{n}}\right| A} & =P_{\left|: x_{1} \ldots x_{n}\right| A}^{n+1}\left(t, t_{1}, \ldots, t_{n}\right)
\end{array}
$$

Lemma 5.2 $\overline{A[s / x]}=\bar{A}[s / x]$, for all formulas $A \in \mathcal{L}$.

Proof. By induction on $A$.

- $\overline{P\left(t_{1}, \ldots, t_{n}\right)[s / x]}=\overline{P\left(t_{1}[s / x], \ldots, t_{n}[s / x]\right)}=P\left(t_{1}[s / x], \ldots, t_{n}[s / x]\right)=$ $P\left(t_{1}, \ldots, t_{n}\right)[s / x]=\overline{P\left(t_{1}, \ldots, t_{n}\right)}[s / x]$

- Let $y \neq x$ and $y \neq s . \overline{(\forall y B)[s / x]}=\overline{\forall y(B[s / x])}=\forall y \overline{(B[s / x])}=$ $\forall y(\bar{B}[s / x])=(\forall y \bar{B})[s / x])=\overline{\forall y B}[s / x]$

The other cases relative to quantified formulas are similar.

- $\overline{\left(\left|t::_{x_{1}}^{t_{1}} \ldots{ }_{x_{n}}^{t_{n}}\right| B\right)[s / x]}=\overline{\left(\left|t[s / x]::_{x_{1}}^{t_{1}[s / x]} \ldots{ }_{x_{n}}^{t_{n}[s / x]}\right| B\right)}=$

$$
\begin{aligned}
& P_{\left|: x_{1} \ldots x_{n}\right| B}^{n+1}\left(t[s / x], t_{1}[s / x], \ldots, t_{n}[s / x]\right)= \\
& \left(P_{\left|: x_{1} \ldots x_{n}\right| B}^{n+1}\left(t, t_{1}, \ldots, t_{n}\right)\right)[s / x]=\overline{\left(\mid t::_{x_{1}}^{t_{1}} \ldots \text { t. }_{x_{n}}^{t_{n}} \mid B\right)}[s / x]
\end{aligned}
$$

We now define a classical theory $\overline{Q . K}_{e}$ whose specific axioms are 


$$
\left\{\bar{A}: Q \cdot K_{e} \vdash A\right\}
$$

Lemma 5.3 $\quad X \vdash_{Q . K_{e}} A \quad$ iff $\quad \bar{X} \vdash{\overline{Q . K_{e}}}_{\bar{A}} \bar{A}$.

Proof. We show that $\vdash_{Q . K_{e}} B_{1} \wedge \ldots \wedge B_{n} \rightarrow A$ iff $\vdash_{\overline{Q . K}_{e}} \overline{B_{1} \wedge \ldots \wedge B_{n} \rightarrow A}$, where $B_{1}, \ldots, B_{n} \in X$.

$\Rightarrow$ holds by definition of $\overline{Q . K}_{e}$

$\Leftarrow$ holds because the specific axioms of $\overline{Q . K}_{e}$ are the translations of the theorems of $Q . K_{e}$ and the inference rules of $\overline{Q . K_{e}}$ are also inference rules of $Q \cdot K_{e}$.

Let $\mathcal{S}$ be a family of classical models for $\overline{Q . K}_{e}$. Each model $w$ is a pair $\left\langle D_{w}, I_{w}\right\rangle$ where $D_{w}$ is a not-empty set, the domain of $w$ and $I_{w}$ is an interpretation function. With $\langle\sigma, w\rangle \models^{c} B$ we denote that the formula $B$ is satisfied by the assignment $\sigma$ in the model $w$ according to the standard classical definition and with $w \models^{c} B$ that $B$ is (classically) true in the model $w$.

Lemma 5.4 Let $\sigma$ be a w-assignment and $A$ a wff of $\mathcal{L}$.

$$
\langle\sigma, w\rangle \models \overline{A[s / x]} \quad \text { iff } \quad\left\langle\sigma^{x \triangleright \sigma(s)}, w\right\rangle \models^{c} \bar{A}
$$

Proof By induction on $A$. We examine the case when $A$ is $\left|t:{ }_{y_{1}}^{t_{1}} \ldots \ldots y_{n}\right| C$.

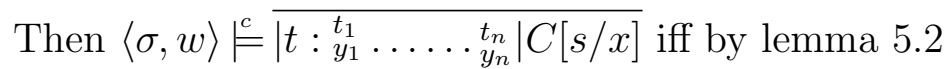

$$
\begin{aligned}
& \langle\sigma, w\rangle \models \overline{|c|:_{y_{1}}^{t_{1}} \ldots \ldots \text { th }_{y_{n}} \mid C}[s / x] \text { iff } \\
& \langle\sigma, w\rangle \models^{c}\left(P_{\left|: y_{1} \ldots y_{n}\right| C}^{n+1}\left(t, t_{1}, \ldots, t_{n}\right)\right)[s / x] \text { iff by lemma } 3.5
\end{aligned}
$$$$
\left\langle\sigma^{x \triangleright \sigma(s)}, w\right\rangle \models^{c} P_{\left|: y_{1} \ldots y_{n}\right| C}^{n+1}\left(t, t_{1}, \ldots, t_{n}\right) \text { iff }
$$$$
\left\langle\sigma^{x \triangleright \sigma(s)}, w\right\rangle \models \overline{=} \overline{\left|t: t_{y_{1}}^{t_{1}} \ldots \ldots y_{n}\right| t_{n} \mid C}
$$

Definition 5.5 Let $w, v$ be $\overline{Q \cdot K_{e}}$-models. For any $a \in D_{w}$ we say that

$$
a \prec v \quad \text { iff } \quad v \models^{c}\left\{\bar{A}:\left\langle\sigma^{x \triangleright a}, w\right\rangle \models^{c} \overline{|x:| A}\right\}
$$

In words, $v$ is compatible with the epistemic state of a iff every sentence known by $a$ is true in $v$. 
Definition 5.6 Let $w, v$ be $\overline{Q . K_{e}}$-models. For any $a \in D_{w}$, a relation $\stackrel{a}{\longmapsto}$ $\subseteq D_{w} \times D_{v}$ is said to be a transition relation admissible for a iff for every $k \geq 0$, every $w$-assignment $\sigma$ and every $v$-assignment $\tau$,

$$
\left\langle\sigma\left(x_{i}\right), \tau\left(x_{i}\right)\right\rangle \in \stackrel{a}{\longmapsto} \quad \text { for } i=1, \ldots, k^{1}
$$

only if

$$
\left\langle\sigma^{x \triangleright a}, w\right\rangle \models^{c} \overline{\left|x: x_{1} \ldots x_{k}\right| A} \quad \Rightarrow \quad\langle\tau, v\rangle \models^{c} \bar{A}
$$

holds for every formula $A$ containing (at most) the variables $x_{1}, \ldots, x_{k}$.

In words, if $\tau\left(x_{i}\right)$ is a counterpart of $\sigma\left(x_{i}\right), 1 \leq i \leq k$, according to $a$, then if $a$ knows of $\sigma\left(x_{1}\right) \ldots \sigma\left(x_{k}\right)$ that $A$, then $A$ is satisfied in $v$ by $\tau\left(x_{1}\right) \ldots \tau\left(x_{k}\right)$.

Lemma 5.7 Let $w$ be a $\overline{Q . K_{e}}$-model and $\langle\sigma, w\rangle \chi^{c}=\overline{\left|x: x_{1} \ldots x_{m}\right| A}$ for some formula $\left|x: x_{1} \ldots x_{m}\right| A$ and $w$-assignment $\sigma$. Then there is a $\overline{Q . K_{e}}$-model $v$ and a $v$-assignment $\tau$ such that:

1. $\langle\tau, v\rangle \ell^{\complement} \bar{A}$;

2. $\sigma(x) \prec v$;

3. the set $\stackrel{\sigma(x)}{\longmapsto}=\left\{\left\langle\sigma\left(x_{1}\right), \tau\left(x_{1}\right)\right\rangle,\left\langle\sigma\left(x_{2}\right), \tau\left(x_{2}\right)\right\rangle, \ldots,\left\langle\sigma\left(x_{m}\right), \tau\left(x_{m}\right)\right\rangle\right\}$ is a transition relation admissible for $\sigma(x)$.

\section{Proof}

- Let $\Gamma$ be the following set of (classical) formulae:

$\Gamma=\{\neg \bar{A}\} \cup\left\{\bar{B}:\langle\sigma, w\rangle \models|c| \overline{\left|x: x_{j_{1}} \ldots x_{j_{h}}\right| B}\right.$, where $\left.\left\{x_{j_{1}} \ldots x_{j_{h}}\right\} \subseteq\left\{x_{1}, \ldots, x_{m}\right\}\right\}$.

First we show that $\Gamma$ is $\overline{Q . K_{e}}$-consistent. Assume by reductio that it is not, then:

(1) $\vdash \overline{Q . K_{e}} \overline{B_{1}} \wedge \ldots \wedge \overline{B_{r}} \rightarrow \bar{A}$

(2) $\vdash_{Q . K_{e}} B_{1} \wedge \ldots \wedge B_{r} \rightarrow A$

(3) $\vdash_{Q . K_{e}}\left|x: x_{1} \ldots x_{m}\right| B_{1} \wedge \ldots \wedge\left|x: x_{1} \ldots x_{m}\right| B_{r} \rightarrow\left|x: x_{1} \ldots x_{m}\right| A$

(4) $\vdash_{Q . K_{e}}\left|x: x_{j_{1}} \ldots x_{j_{h_{1}}}\right| B_{1} \wedge \ldots \wedge\left|x: x_{j_{1}} \ldots x_{j_{h_{r}}}\right| B_{r} \rightarrow\left|x: x_{1} \ldots x_{m}\right| A$

(5) $\vdash \overline{Q . K_{e}} \overline{\left|x: x_{j_{1}} \ldots x_{j_{h_{1}}}\right| B_{1}} \wedge \ldots \wedge \overline{\left|x: x_{j_{1}} \ldots x_{j_{h_{r}}}\right| B_{r}} \rightarrow \overline{\left|x: x_{1} \ldots x_{m}\right| A}$

Therefore, we would have that $\langle\sigma, w\rangle|c| \overline{\left|x: x_{1} \ldots x_{m}\right| A}$ contrary to the fact that $\langle\sigma, w\rangle \ell^{\in}=\overline{\left|x: x_{1} \ldots x_{m}\right| A}$.

Since $\Gamma$ is $\overline{Q . K_{e}}$-consistent, by classical model theory there is a model $v$ and a $v$-assignment $\tau$ such that $\left.\langle\tau, v\rangle\right|^{c} \Gamma$, therefore $\langle\tau, v\rangle \ell^{c} \bar{A}$.

${ }^{1}$ We also write $\sigma\left(x_{i}\right) \stackrel{a}{\longmapsto} \tau\left(x_{i}\right) \quad$ for $i=1, \ldots, k$ 
- By the way $\Gamma$ is defined, $\Gamma$ contains all the formulae $\bar{B}$ without free variables such that $\langle\sigma, w\rangle \models|c|=|x:| B$, therefore $\sigma(x) \prec v$.

- We have to show that the set $\stackrel{\sigma(x)}{\longmapsto}$ is a counterpart relation admissible for $\sigma(x)$, i.e. for any $k>0$, any formula $C\left(y_{1}, \ldots, y_{k}\right)$, any $w$-assignment $\pi$ and any $v$-assignment $\mu$, if

$$
\begin{gathered}
\text { (i) } \pi(y)=\left.\sigma(x) \quad(i i) \quad\langle\pi, w\rangle\right|^{c} \overline{\left|y: y_{1} \ldots y_{k}\right| C} \\
\text { (iii) } \pi\left(y_{i}\right) \stackrel{\sigma(x)}{\longmapsto} \mu\left(y_{i}\right) \quad i=1 \ldots k
\end{gathered}
$$

then

$$
\langle\mu, v\rangle \models \overline{C\left(y_{1}, \ldots, y_{k}\right)}
$$

By the definition of $\stackrel{\sigma(x)}{\longmapsto}$, if $\pi\left(y_{i}\right) \stackrel{\sigma(x)}{\longmapsto} \mu\left(y_{i}\right), i=1 \ldots k$, then for some $x_{j_{i}} \in\left\{x_{1}, \ldots, x_{m}\right\}$,

$$
\pi\left(y_{i}\right)=\sigma\left(x_{j_{i}}\right)
$$

and

$$
\mu\left(y_{i}\right)=\tau\left(x_{j_{i}}\right)
$$

It follows from $(i i)$ that:

$$
\left\langle\pi^{y_{1} \triangleright \sigma\left(x_{j_{1}}\right) \ldots y_{k} \triangleright \sigma\left(x_{j_{k}}\right)}, w\right\rangle \models \overline{\left|y: y_{1} \ldots y_{k}\right| C}
$$

Given that $y_{1}, \ldots, y_{k}$ are all the free variables in $\bar{C}$ and that $\pi(y)=\sigma(x)$, this is equivalent to:

$$
\left.\left\langle\sigma^{y \triangleright \sigma(x), y_{1} \triangleright \sigma\left(x_{j_{1}}\right) \ldots y_{k} \triangleright \sigma\left(x_{j_{k}}\right)}, w\right\rangle\right|^{c} \overline{\left|y: y_{1} \ldots y_{k}\right| C}
$$

By lemma 3.5 we get that:

$$
\left.\langle\sigma, w\rangle\right|^{c} \overline{\left|y[x / y]: y_{j_{1}}^{x_{j_{1}} \ldots y_{k}} x_{j_{k}}\right| C}
$$

Then by MP with the (translation of the) axiom $R G^{v}$ it obtains that:

$$
\langle\sigma, v\rangle \models \overline{\left|x: v_{1} \ldots v_{h}\right|\left(C\left[x_{j_{1}} / y_{1} \ldots x_{j_{k}} / y_{k}\right]\right)}
$$

Given that $\left\{v_{1}, \ldots, v_{h}\right\} \subseteq\left\{x_{j_{1}}, \ldots, x_{j_{k}}\right\} \subseteq\left\{x_{1}, \ldots, x_{m}\right\}$, it follows that $\overline{C\left[x_{j_{1}} / y_{1} \ldots x_{j_{k}} / y_{k}\right]} \in \Gamma$. Therefore

$$
\langle\tau, v\rangle \models \bar{C} \overline{C\left[x_{j_{1}} / y_{1} \ldots x_{j_{k}} / y_{k}\right]}
$$


By lemma 3.5 we get that:

$$
\left\langle\tau^{y_{1} \triangleright \tau\left(x_{j_{1}}\right) \ldots y_{k} \triangleright \tau\left(x_{j_{k}}\right)}, v\right\rangle \models \overline{C\left(y_{1}, \ldots, y_{k}\right)}
$$

But all the free variables of $\bar{C}$ are among $y_{1}, \ldots, y_{k}$, therefore this is equivalent to:

$$
\left\langle\mu^{y_{1} \triangleright \tau\left(x_{j_{1}}\right) \ldots y_{k} \triangleright \tau\left(x_{j_{k}}\right)}, v\right\rangle \models^{c} \overline{C\left(y_{1}, \ldots, y_{k}\right)}
$$

By the definition of $\stackrel{\sigma(x)}{\longmapsto}$, if $\pi\left(y_{i}\right) \stackrel{\sigma(x)}{\longmapsto} \mu\left(y_{i}\right)$ for all $i=1 \ldots k$, then, for all $i=1 \ldots k$ there is a $x_{j_{i}} \in\left\{x_{i}, \ldots, x_{m}\right\}$ such that $\tau\left(x_{j_{i}}\right)=\mu\left(y_{i}\right)$. Therefore we have:

$$
\left.\left\langle\mu^{y_{1} \triangleright \mu\left(y_{1}\right) \ldots y_{k} \triangleright \mu\left(y_{k}\right)}, v\right\rangle\right|^{c} \overline{C\left(y_{1}, \ldots, y_{k}\right)}
$$

i.e.

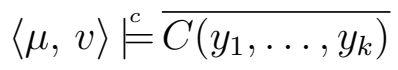

The set $\stackrel{\sigma(x)}{\longmapsto}$ as defined in lemma 5.7 gives the minimal counterpart relation that links the model $w$ to the model $v$ in dependence of the formula $A$, the $w$-assignment $\sigma$ and the individual $\sigma(x)$. Between $D_{w}$ and $D_{v}$ no other counterpart relation is taken into account even if extensions of $\stackrel{\sigma(x)}{\longmapsto}$ may be admissible. If $\sigma(x)=a$ for some $a \in D_{w}$, we call the set $\stackrel{a}{\longmapsto}$ the canonical counterpart relation relative to $a, w$ and $v$, in brief $\operatorname{CNTP}(a, w, v)$. Notice that if $\operatorname{CNTP}(a, w, v) \neq \emptyset$, then $a \prec w$.

Definition 5.8 Let $\mathcal{S}$ be a set of $\overline{Q . K_{e}}$-models. We say that:

- $w \in \mathcal{S}$ is realized in $\mathcal{S}$ iff for each $w$-assignment $\sigma$ and each formula $\left|x: x_{1} \ldots x_{m}\right| A$ of $\mathcal{L}$, if $\langle\sigma, w\rangle$ 作 $\overline{\left|x: x_{1} \ldots x_{m}\right| A}$, then there is a $\overline{Q . K_{e}}$ model $v \in \mathcal{S}$ and a $v$-assignment $\tau$ such that:

$$
\begin{aligned}
& -\sigma(x) \prec v ; \\
& -\sigma\left(x_{i}\right) \stackrel{\sigma(x)}{\longmapsto} \tau\left(x_{i}\right), \text { for every } x_{i} \in\left\{x_{1}, \ldots, x_{m}\right\} ; \\
& -\langle\tau, v\rangle \not^{\in} \bar{A} .
\end{aligned}
$$

- $\mathcal{S}$ is fully realized iff every member of $\mathcal{S}$ is realized in $\mathcal{S}$ and for any $z, w \in \mathcal{S}$, if $z \neq w$ then $D_{z} \cap D_{w}=\emptyset$.

Lemma 5.9 For every $\overline{Q . K_{e}}$-model $w$ there is a set $\mathcal{S}^{w}$ of $\overline{Q . K_{e}}$-models such that: 
- $w \in \mathcal{S}^{w}$

- $\mathcal{S}^{w}$ is fully realized.

PROOF We define a chain $\mathcal{S}_{0}, \mathcal{S}_{1} \ldots, \mathcal{S}_{n}, \ldots$ of sets of classical models such that $\mathcal{S}_{0}=\{w\}$ and $\mathcal{S}_{n+1}$ is obtained fron $\mathcal{S}_{n}$ by adding to it new $\overline{Q . K_{e}}$-models so as to realize the models already present in $\mathcal{S}_{n}$. This step is performed according to lemma 5.7 taking care to choose models whose domains do not overlap the domains of the models already present in $\mathcal{S}_{n}$. Let $\mathcal{S}^{w}$ be the union of the chain.

The fully realized set $\mathcal{S}^{w}$ whose elements are constructed according to lemma 5.7 is said to be canonical. In a canonical (fully realized) set the relation $\operatorname{CNTP}(a, w, v)$ is uniquely determined given $w$ and $v$, in fact if $\operatorname{CNTP}(a, w, v)=C N T P(b, w, v)$ then $a=b$, so as far as canonical sets are concerned, we will talk of the relation $C N T P(w, v)$.

Given a canonical set $\mathcal{S}^{w}$, the model $\mathcal{M}^{\mathcal{S}^{w}}=\left\langle\mathcal{S}^{w}, \mathcal{D}, \prec, \longmapsto, \mathcal{I}\right\rangle$ is said to be a canonical epistemic model if

- $\mathcal{D}$ is a function such that for every $z=\left\langle D_{z}, I_{z}\right\rangle \in \mathcal{S}^{w}, D(z)=D_{z}$

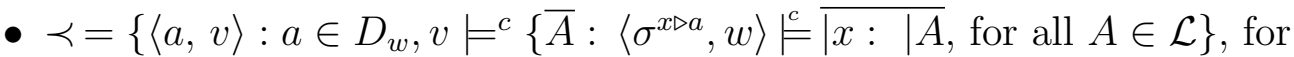
some $w, v \in \mathcal{S}^{w}$, and $w$-assignment $\left.\sigma\right\}$

- $\longmapsto=\bigcup\{C N T P(w, v)\}_{w, v \in S^{w}}$

- $\mathcal{I}$ is a function such that for every $z=\left\langle D_{z}, I_{z}\right\rangle \in \mathcal{S}^{w}, I(z)=I_{z}$

Lemma 5.10 Given a canonical epistemic model $\mathcal{M}^{\mathcal{S}^{w}}=\left\langle\mathcal{S}^{w}, \mathcal{D}, \prec, \longmapsto\right.$ , $\mathcal{I}\rangle$, for every formula $B$ of $\mathcal{L}$ and every z-assignment $\sigma$,

$$
\sigma \models=_{z}^{\mathcal{M}^{\mathcal{S}^{w}}} B \quad \text { iff } \quad\langle\sigma, z\rangle \models \bar{B}
$$

for all $z \in \mathcal{S}^{w}$.

PROOF By induction on $B$. We examine just two cases.

- If $B$ is atomic, the lemma holds thanks to the definition of the interpretation function $\mathcal{I}$ of $\mathcal{M}^{\mathcal{S}^{w}}$.

- $B=\left|t:{ }_{y_{1}}^{t_{1}} \ldots{ }_{y_{n}}^{t_{n}}\right| C$, where $f v(B)=\left\{y, y_{1}, \ldots, y_{n}\right\}$.

If $\sigma \not \forall_{z}^{\mathcal{M}}\left|t::_{y_{1}}^{t_{1}} \ldots{ }_{y_{n}}^{t_{n}}\right| C$, then by lemma 3.5

$$
\sigma^{y \triangleright \sigma(t), y_{1} \triangleright \sigma\left(t_{1}\right), \ldots y_{n} \triangleright \sigma\left(t_{n}\right)} \forall_{z}^{\mathcal{M}^{\mathcal{S}^{w}}}\left|y: y_{1} \ldots y_{n}\right| C
$$


where $y$ is a variable different from $y_{1} \ldots y_{n}$.

To simplify the notation, let $\pi=\sigma^{y \triangleright \sigma(t), y_{1} \triangleright \sigma\left(t_{1}\right), \ldots y_{n} \triangleright \sigma\left(t_{n}\right)}$, then

$$
\pi \forall_{z}^{\mathcal{M}^{\mathcal{S}^{w}}}\left|y: y_{1} \ldots y_{n}\right| C
$$

By definition 3.3 of satisfaction there is a $v$ such that $\pi(y) \prec v$, a $v$-assignment $\tau$ such that $\tau \forall_{v}^{\mathcal{M}^{\mathcal{S}^{w}}} C$, and moreover $\pi\left(y_{i}\right) \stackrel{\pi(y)}{\longmapsto} \tau\left(y_{i}\right), 1 \leq i \leq n$. By induction hypothesis $\langle\tau, v\rangle \ell_{\models}=\bar{C}$. Since $\pi\left(y_{i}\right) \stackrel{\pi(y)}{\longmapsto} \tau\left(y_{i}\right), 1 \leq i \leq n$,

$$
\langle\pi, z\rangle \ell^{£} \overline{\left|y: y_{1} \ldots y_{n}\right| C}
$$

thanks to the definition 5.6. Consequently $\langle\sigma, z\rangle \chi_{\models}=\overline{\left|t:{ }_{y_{1}}^{t_{1}} \ldots{ }_{y_{n}}^{t_{n}}\right| C}$ by lemma 3.5 and the definition of $\pi$.

Conversely, if $\langle\sigma, z\rangle \not \models=\overline{\left|t: t_{y_{1}}^{t_{1}} \ldots \ldots y_{n}\right| C}$, then by definition 2.3,

$$
\langle\sigma, z\rangle \ell^{\in} \overline{\left|y: y_{1} \ldots y_{n}\right| C\left[t / y, t_{1} / y_{1} \ldots t_{n} / y_{n}\right]}
$$

where $y$ is a variable different from $y_{1} \ldots y_{n}$, hence by lemma 5.4

$$
\left.\left\langle\sigma^{y \triangleright \sigma(t), y_{1} \triangleright \sigma\left(t_{1}\right) \ldots \ldots y_{n} \triangleright \sigma\left(t_{n}\right)}, z\right\rangle\right\rangle_{\models}=\overline{\left|y: y_{1} \ldots y_{n}\right| C}
$$

To simplify the notation, let $\pi=\sigma^{y \triangleright \sigma(t), y_{1} \triangleright \sigma\left(t_{1}\right), \ldots y_{n} \triangleright \sigma\left(t_{n}\right)}$, then

$$
\langle\pi, z\rangle \ell_{\models}=\overline{\left|y: y_{1} \ldots y_{n}\right| C}
$$

Since $\mathcal{S}^{w}$ is fully realized, there is a classical model $v$ such that $\pi(y) \prec v$ and there is a $v$-assignment $\tau$ such that $\left.\langle\tau, v\rangle\right|^{c}\left\{\bar{B}:\left.\langle\pi, z\rangle\right|^{c} \overline{\left|y: y_{1} \ldots y_{n}\right| B}\right\} \cup$ $\{\neg \bar{C}\}$ and moreover $\pi\left(y_{i}\right) \stackrel{\pi(y)}{\longmapsto} \tau\left(y_{i}\right), 1 \leq i \leq n$. Hence $\langle\tau, v\rangle \models_{v} \neg \bar{C}$, $\langle\tau, v\rangle \not \forall_{v} \bar{C}$, therefore by induction hypothesis $\tau \forall_{v}^{\mathcal{M}^{\mathcal{S}^{w}}} C$. Since $\pi\left(y_{i}\right) \stackrel{\pi(y)}{\longmapsto}$ $\tau\left(y_{i}\right), 1 \leq i \leq n, \pi \forall_{z}^{\mathcal{M}^{\mathcal{S}^{w}}}\left|y: y_{1} \ldots y_{n}\right| C$ by definition 5.6. Consequently $\sigma \forall_{z}^{\mathcal{M}^{\mathcal{S}^{w}}}\left|t::_{y_{1}}^{t_{1}} \ldots y_{y_{n}}^{t_{n}}\right| C$.

Theorem 5.11 (Completeness) If a wff $A \in \mathcal{L}$ is not a theorem of Q.K $K_{e}$, then it is not valid on the class of transition epistemic models.

\section{Correspondence}

- What is KnOWn is true $\left(T_{e}\right) \quad\left|x: x_{1} \ldots x_{n}\right| A \rightarrow A$

It corresponds to the following conditions: 
$-a \in D_{w}$ only if $a \prec w$

- for all $a, b \in D_{w}, b \stackrel{a}{\longmapsto} b$

Let $a=\sigma(y)$ for some $y$ and $\sigma$. If axiom $T_{e}$ holds, then $\left.w\right|^{c}\{\bar{A}$ : $\left.\left.\langle\sigma, w\rangle\right|^{c} \overline{|y:| A}\right\}$, therefore $a \prec w$. Moreover, since $\langle\sigma, w\rangle \models \overline{\mid} \overline{\left|t: x_{1} \ldots x_{n}\right| A}$ only if $\langle\sigma, w\rangle \models \bar{\models} \bar{A}$, then $\sigma\left(x_{i}\right) \stackrel{\sigma(t)}{\longmapsto} \sigma\left(x_{i}\right)$

- Positive introspection $\quad\left(4_{e}\right) \quad\left|x: x_{1} \ldots x_{n}\right| A \rightarrow\left|x: x, x_{1} \ldots x_{n}\right| \mid x$ : $x_{1} \ldots x_{n} \mid A$

It corresponds to the following conditions:

- given $a \in D_{w}$ and $b \in D_{v}$, if $a \stackrel{a}{\longmapsto} b$ and $b \prec z$, then $a \prec z$

- for all $a, b \in D_{w}, c, d \in D_{v}, e \in D_{z}$, if $a \stackrel{a}{\longmapsto} d$ and $b \stackrel{a}{\longmapsto} c$ and $c \stackrel{d}{\longmapsto} e$, then $a \stackrel{a}{\longmapsto} e$

- Negative introspection

$\left(5_{e}\right) \quad \neg\left|x: x_{1} \ldots x_{n}\right| A \rightarrow\left|x: x, x_{1} \ldots x_{n}\right| \neg \mid x:$ $x_{1} \ldots x_{n} \mid A$

It corresponds to the following conditions:

- given $a \in D_{w}$ and $b \in D_{z}$, if $a \prec v, a \prec z$ and $a \stackrel{a}{\longmapsto} b$, then $b \prec v$

- for all $a, d \in D_{w}, c, b \in D_{v}, e \in D_{z}$, if $d \stackrel{a}{\longmapsto} c$ and $d \stackrel{a}{\longmapsto} e$ and $a \stackrel{a}{\longmapsto} b$ then $c \stackrel{b}{\longmapsto} e$

As shown in [2], some conditions of the counterpart relation correspond to modal formulas.

- The Barcan formula : $\forall y\left|x: y, x_{1}, \ldots, x_{n}\right| A \rightarrow\left|x: x_{1}, \ldots, x_{n}\right| \forall y A$ corresponds to the property of the counterpart relation of being surjective.

If Peter knows of all his friends that they are trustworthy, then Peter knows that all his friends are trustworthy. 
$\forall y(\operatorname{Best}$ Friend $(y$, Peter $) \rightarrow \mid$ Peter $: y \mid$ Trustworthy $(y)) \rightarrow \mid$ Peter $:$ $\mid \forall y(\operatorname{Best}$ Friend $(y$, Peter $) \rightarrow \operatorname{Trustworthy}(y))$

This sentence can be falsified if in worlds compatible with the epistemic state of Peter now, Peter has friends apart from the Peter-counterparts of his friends now.

- The Ghilardi formula : $\exists y\left|x: y, x_{1}, \ldots, x_{n}\right| A \rightarrow\left|x: x_{1}, \ldots, x_{n}\right| \exists y A$ corresponds to the property of the counterpart relation of being everywhere defined.

If Peter knows of his best friend that he is trustworthy, then Peter knows that someone is trustworthy.

$\exists y(\operatorname{BeSt} \operatorname{Friend}(y$, Peter $) \wedge \mid$ Peter $: y \mid$ Trusthworthy $(y)) \rightarrow \mid$ Peter : $\exists y$ TRUSTWORTHY $(y)$

This sentence can be falsified if in worlds compatible with the epistemic state of Peter now, there are no Peter-counterparts of Peter's best friend now.

- The Knowledge of identity : $x=y \rightarrow|z: x, y|(x=y)$ corresponds to the property of the counterpart relation of being functional.

If Peter's best friend is Brian's father, then Peter knows of his best friend that he is Brian's father.

$P^{\prime} b f=B^{\prime} f \rightarrow \mid$ Peter $:{ }_{x}^{P^{\prime} b f},{ }_{y}^{B^{\prime} f} \mid(x=y)$

This sentence can be falsified if in worlds compatible with the epistemic state of Peter now, Peter-counterparts of Peter's best friend now are different from Peter-counterparts of Brian's father.

- The Knowledge of Diversity : $x \neq y \rightarrow|z: x, y|(x \neq y)$ corresponds to the property of the counterpart relation of not being convergent.

If Peter's best friend is not Brian's father, then Peter knows of his best friend that he is not Brian's father.

$P^{\prime} b f \neq B^{\prime} f \rightarrow\left|p:{ }_{x}^{P^{\prime} b f},{ }_{y}^{B^{\prime} f}\right|(x \neq y)$

This sentence can be falsified since Peter-counterparts of Peter's best friend now can be the same as Peter-counterparts of Brian's father in all worlds compatible with the epistemic state of Peter now. 


\section{References}

[1] Brä̈ner, Torben \& Ghilardi, Silvio, "First-Order Modal Logic ", in Patrick Blackburn, Johan van Benthem, Frank Wolter (eds), Handbook of Modal Logic, Elsevier, 2007,

[2] Corsi, Giovanna, "Necessary for", in Clark Glymor, Wang Wei and Dag Westerståhl (eds), Logic Methodology and Philosophy of Science, Proceedings of the Thirtheen International Congress, King's College Publications, 2010, pp. 162-184.

[3] Corsi, Giovanna \& TAssi, Gabriele, "Agenti e Transizioni", presented at Incontro di Logica in onore di Annalisa Marcja, University of Florence, Department of Mathematics, 6-7 May 2010.

[4] Fagin, Ronald, Halpern, Joseph Y., Moses, Yoram and Vardi, Moshe Y., Reasoning about Knowledge The MIT Press, 1995.

[5] Halpern, Joseph Y., Moses, Yoram, "A Guide to Completeness and Complexity for Modal Logics of Knowledge and Belief", Artificial Intelligence 54, 1992, pp. 319-379.

[6] Hintikka, Jaakko, Knowledge and Belief. An Introduction to the Logic of the two Notions Cornell University Press, 1962.

[7] TAssi, Gabriele, Cone Transition Semantics, forthcoming. 